\title{
Apuntes sobre diversidad cultural, interculturalidad e interdisciplinariedad
}

\author{
Notes about Diversity, Interculturality and Interdisciplinarity
}

\author{
Mahia Maurial MacKee ${ }^{1}$
}

\section{Resumen}

La autora desarrolla el concepto de la diversidad peruana cultural, lingüística y de género; reflexiones sobre el tema de la educación superior y la diversidad en las aulas y los logros con la experiencia en educación intercultural bilingüe; $y$, los retos de la interculturalidad universitaria, tal que se fortalezcan la interdisciplinariedad.

Palabras claves: diversidad, interculturalidad, educación superior.

\begin{abstract}
The author develops the concept of Peruvian cultural, linguistic and gender diversity; reflections on the theme of higher education and diversity in the classroom and achievements with experience in bilingual intercultural education; and, the challenges for university interculturality, such as strengthening interdisciplinarity.
\end{abstract}

Keywords: diversity, interculturality, higher education.

\section{Introducción}

En este artículo $^{1}$ desarrollamos primero el concepto de diversidad para centrarnos luego en la diversidad cultural, lingüística, biológica y de género (relaciones entre el hombre y la mujer). En la segunda parte reflexionamos sobre el tema de la educación superior y la diversidad existente en las aulas y los logros que implica la experiencia en Educación Intercultural Bilingüe en nuestra universidad. En la tercera y última parte, planteamos algunos retos para "interculturalizar" la universidad desde los estudios ambientales y hacia el desarrollo sostenible, donde se abra el espectro hacia un diálogo de saberes y se fortalezca la interdisciplinariedad.

1 Algunas ideas desarrolladas aquí sobre la diversidad las escribí en el marco referencial del "Proyecto de investigación "Discursos y prácticas pedagógicas frente a la diversidad estudiantil de aulas multigrado de primaria de Tacna y Ayacucho", de la Facultad de Educación de la UPCH, donde participaron la Dra. Olga Bardales y la Lic. Rosa Alonso, además de mi persona. Otras fueron pensadas y hoy digeridas como parte de mi práctica laboral, de profesora en aulas de Educación Intercultural Bilingüe y en el trabajo de campo con comunidades campesinas y nativas en el Perú, lo que alimentó mi tesis doctoral. Además, las ideas de la última parte de este artículo fueron desarrolladas en mi exposición del V Seminario Nacional de la Sociedad de Investigación Educativa Peruana, realizado en Ayacucho en noviembre de 2016. Gracias al Dr. Manuel Bello por impulsarme a escribir el artículo, y al Mg. Renato Merino y a la Lic. Ingrid Guzmán por comentarlo. 


\section{Conceptualizando la diversidad y sus formas más recurrentes}

La noción de diversidad cultural según UNESCO (2001), en su Artículo $1^{\circ}$ implica:

Fuente de intercambios, de innovación y de creatividad, la diversidad cultural es, para las personas, tan necesaria como la diversidad biológica para los organismos vivos. En este sentido, constituye la herencia común de la humanidad y debe ser reconocida y asegurada en beneficio de las generaciones presentes y futuras (UNESCO, 2001, p. 4)

Para UNESCO (2001), la diversidad es patrimonio de la humanidad y debe ser conservada; en otras palabras, es una potencialidad para el mundo, así como para la realidad de la sociedad y la educación peruana.

Diversidad lingüistica. En el Informe de Unesco "Nuestra Diversidad creativa", aparecido en 1996, ad portas del siglo XXI, se aplaude por primera vez con solidez y de manera sistemática en las Naciones Unidas la diversidad cultural -heterogéneo acervo de prácticas, cosmovisiones y valores de un pueblo o grupo humano- y lingüística del planeta, y se considera a la última como parte de la primera. De hecho, la existencia de lenguas heterogéneas y diversas son parte del acervo de la cultura de un pueblo y la contienen. El problema, como señala el documento recién citado, (UNESCO, 1996), es que la tendencia globalizadora de las últimas décadas en el planeta, si bien favorable en algunos sentidos -como la intensa interconexión, comunicación e información-, apunta hacia la homogeneización y la desaparición de las lenguas, sobre todo de las minorías indígenas. Por ello resulta clave promover la implementación de la Educación Intercultural Bilingüe (EIB) (bilingüe en castellano y en una lengua originaria), carrera de la Facultad de Educación de esta casa de estudios, sobre la que tratamos más adelante.
En el Perúexisten hablantes de 47 lenguas originarias, 20 con alfabetos oficiales, 4 de ellas andinas (siendo las principales el quechua con sus 6 grupos 24 variedades y el aimara) y 43 lenguas amazónicas, (MINEDU, 2013), además de la lingua franca, el español, y varias lenguas extranjeras incorporadas (inglés, francés, italiano, chino mandarín, japonés y otras). Todas estas lenguas constituyen un valioso recurso de diversidad lingüística.

Relación entre la diversidad biológica y la diversidad cultural y lingüistica. La diversidad biológica se relaciona con la diversidad cultural y lingüística, en tanto, la domesticación y el cultivo de los tubérculos menores (ollucos, mashuas y ocas por ejemplo), acaecidos por milenios $-\mathrm{y}$ desplazados en nuestro país "hacia la oscuridad" durante la Conquista (National Research Council, 1989)- ha significado la dación de nombres en quechua o aimara para estos y la creación de procesos agrícolas, así como la elaboración de recetas gastronómicas, rituales y usos para cuidados de la salud con esas variedades de tubérculos. Sin contar con las raíces o granos, legumbres, vegetales frutas o nueces domesticados en la región andina (National Research Council, 1989).

Diversidad de género. Para comunicar sobre diversidad de género, nos centramos en la relación entre hombres y mujeres. Consideremos que sexo es una definición de corte biológico, mientras que género es una construcción sociocultural que depende de las pautas de crianza y los roles relacionados con lo femenino y lo masculino, tal como se dan en la sociedad (Money y Stoller, 1964, citado en Hernández, 2006). UNESCO (1996) ensalza la diversidad de género opuesta a la tendencia patriarcal de favorecer a los varones por sobre las mujeres en el goce y ejercicio de sus derechos, y la inequidad existente entre hombres y mujeres. En el documento de UNESCO (1996) se toma la agenda de Beijing y se da importancia al derecho a la "participación cívica y cultural de la mujer" (p. 30), refiriéndose al ámbito institucional, político y 
profesional. Creemos que ello se forma desde el aula del parvulario, cuando el docente promueve el trato igualitario y la participación equitativa de hombre y mujer o niña y niño (Maurial, 2003a).

La diversidad de género se ve en mayor medida en el Perú en las carreras de humanidades y menos en carreras de las ciencias (en el Perú, una de cada cuatro estudiantes de ciencias son mujeres ${ }^{2}$ ). Hace falta un trabajo de sensibilización y empoderamiento de las mujeres durante su infancia para que se equipare la cuota de género en las ciencias. Ello debe ir a la par de un cambio en la sociedad de roles -menos mujeres "cuidadoras" y más mujeres "científicas"y predilecciones profesionales que superen los estereotipos de género.

El currículum nacional (MINEDU, 2016) plantea un enfoque transversal a la igualdad de género, donde la diversidad sería la concreción y la otra cara de la moneda de la equidad en abstracto (Jelin, 2003, citada en Maurial, 2003). El currículum vigente, en este sentido, reconoce y plantea el tratamiento de género propugnando los valores de igualdad y dignidad, justicia y empatía entre géneros (MINEDU, 2016).

Relación entre diversidad de género y diversidad cultural. Para relacionar estos dos tipos de diversidades es importante situarse en la construcción de un enfoque intercultural -o de diálogo (horizontal) entre culturas- en el que lo académico recoge el discurso feminista proveniente del hemisferio norte, enmarcado en los derechos humanos, pero es a su vez sensible a la construcción social local de género o a las concepciones y performance de lo femenino y/o masculino desde las culturas originarias, si bien en transformación cultural. Es importante contextualizar culturalmente las políticas educativas, de investigación y desarrollo de la diversidad que relacione el género y la cultura, seleccionando las buenas prácticas de cada cultura con respecto a las

2 Véase http://rpp.pe/blog/innovados/cuantas-mujeres-cientificas-hayen-el-peru-noticia-940220 relaciones de género, de tal manera de construir modelos propios. La sociedad civil organizada y las líderes indígenas mujeres tienen mucho que decir al respecto (Maurial, 2003), junto con los funcionarios de Estado y las universidades.

\section{La diversidad existente en nuestras aulas universitarias}

Como este estudio sigue un enfoque cualitativo, se refiere a un caso en específico, que se adscribe a un método inductivo, no generalizable. En la carrera de Educación Intercultural Bilingüe (EIB) de la Facultad de Educación de la Universidad Peruana Cayetano Heredia existen alumnos de Beca 18, hablantes del castellano y de lenguas originarias, las que son en algunos casos su lengua materna y en otros casos su segunda lengua. Los estudiantes también estudian inglés como lengua extranjera, requisito indispensable para su graduación y que los conecta con la Aldea Global y las publicaciones en inglés, que son reiterativamente más avanzadas que las de español. Además, los estudiantes de esta carrera estudian cuatro cursos de castellano y cuatro de lengua originaria, en tanto muchos de ellos tendrán como escenario lingüístico de su comunidad y de sus escuelas uno donde se habla el castellano y una lengua indígena, u otros revitalizarán la lengua originaria de sus abuelos.

En la carrera de EIB se aprovecha la diversidad lingüística presente, a nivel oral, para las exposiciones de todos los cursos -que son en castellano y en lengua originaria- y para la producción de videos por parte de los estudiantes; y, a nivel escrito, para la redacción de relatos, los mismos que contienen importante sabiduría, cosmovisión y valores. También se utiliza la lengua originaria para la hoja informativa del que testimonia, o informante, y en la realización y transcripción de parte de las entrevistas producto de las investigaciones, en los cursos de investigación. El uso de las lenguas originarias en lo académico le da legitimidad a la misma y a los hablantes en un nuevo ámbito que 
tradicionalmente es del castellano en nuestro país. Los usos funcionales de las lenguas originarias se han dado en el hogar o en las comunidades, en el mercado o en la feria por ejemplo (Zúñiga, 1987), y desde las últimas décadas en las escuelas y en los últimos años en las aulas universitarias de EIB. En eta carrera, donde en la especialidad de educación inicial el conjunto está conformado por mujeres y en la especialidad de primaria hay un número similar de hombres y de mujeres, se potencia la participación equitativa tanto de estudiantes mujeres como de varones con las preguntas a los docentes, o dándoles voz en sus comentarios o respuestas; así como en la creación de equipos de trabajo heterogéneos dentro y fuera del aula, que se distribuyen en forma rotativa la coordinación, secretaría y relatoría del grupo, formando líderes hombres y mujeres.

Diversidad de niveles de avance. Los niveles de avance presentes en el aula responden a la heterogeneidad en los aprendizajes de los estudiantes $\mathrm{y}$, por ende, al manejo diferente que hace el docente de los niveles en el aprendizaje de determinadas capacidades o competencias de los estudiantes. El o la docente pueden conformar grupos heterogéneos según el nivel de avance de los estudiantes para que los alumnos regulares aprendan de los buenos alumnos, por ejemplo (Maurial, 2000). Para ello el docente deberá manejar una evaluación en proceso de los y las estudiantes.

Diversidad de recursos para el aprendizaje y la investigación. En las aulas de la carrera de EIB se aprovechan recursos de las nuevas Tecnologías de la Información y la Comunicación; así, por ejemplo, se utiliza el blogfolio (mezcla de portafolio y blog) como recurso de aprendizaje, lo que permite la reflexión del alumnado; y también se utiliza los videos de Youtube para realizar video-foros. Cabe mencionar que se "interculturalizan" los procesos de enseñanza-aprendizaje y los procesos de formas alternativas de recojo de la información, con el uso de las artes (por ejemplo cerámica y dibujo) que son parte del acervo indígena y que se combinan con el uso de tecnologías en el recojo de grabaciones de voz, videos y fotografías por parte de los estudiantes, durante el trabajo de campo o de sus prácticas preprofesionales, los cuales son descritos, transcritos y analizados por ellos mismos. ${ }^{3}$

Además, en investigación los alumnos aprovechan los recursos electrónicos de la Biblioteca Central de la UPCH para la búsqueda de fuentes secundarias y se "interculturalizan" las formas de investigación realizadas en la práctica de campo, entre métodos y técnicas convencionales que se combinan con el recojo de dibujos (ya mencionados) de niños, niñas y padres o madres de familia -que expresan las cosmovisiones indígenas- y relatos de sabios comunales (que conocen sobre técnicas tradicionales en medicina, artesanía, agricultura, etc.). Estos relatos se recogen en la lengua originaria o también en español, en comunidades o pueblos que han atravesado transformaciones culturales.

\section{Retos para interculturalizar la universidad y volverla más interdisciplinaria}

La interculturalidad implica "procesos de negociación, intercambio de saberes y coconstrucción social en valoración del otro" (Abarca, 2015, p. 1). En la carrera de EIB de la Facultad de Educación de la UPCH se ha iniciado un "diálogo de saberes". Ishizawa (2012) lo conceptualiza como "la relación mutuamente enriquecedora entre personas y culturas, puestas en colaboración por un destino compartido" (p.5). Ello implica la creación de lazos entre culturas $y$, específicamente, entre los conocimientos académicos por un lado y los conocimientos de los pueblos indígenas (incluidos saberes de los/las estudiantes y data de campo de fuentes primarias) por otro. Se ha iniciado entonces un diálogo entre conocimientos, tecnologías y recursos que se facilita por la diversidad lingüística y cultural de alumnos y docentes, por la diversidad de

3 Cabe mencionar la danza y la música originaria que se presentaban antes de la pandemia, como parte de las actividades interculturales de la Facultad de Educación, en el Auditorio Central de la UPCH. 
buenas prácticas para el aprendizaje que promueven los últimos, y por el currículo que se implementa en la carrera.

A nivel de saberes, por ejemplo, se tiene en la malla curricular un curso de matemática que es convencional y otro de etnomatemática. Se enseña la didáctica convencional en el curso de didáctica general, pero también se repasan las formas de hacer, pensar y actuar indígenas en los cursos de socialización y subjetividad I y II, o se trabaja el currículo nacional de la educación básica (MINEDU, 2016); y, se conjuga con una pedagogía que parte por trabajar temas en función del calendario comunal y agro-festivo de la comunidad. En los cursos de procesos históricos de los pueblos indígenas I y II se pasa revista a la historia nacional, paralela a procesos indígenas, como la historia del caucho que esclavizó a estos. En suma, se realiza una interculturalidad crítica, enfoque que plantea el currículo en la Facultad de Educación de la UPCH. Esta visión de la interculturalidad intenta "romper con la historia hegemónica de una cultura dominante y otra subordinada -romper con la matriz colonial y su acompañante: el mestizaje como discurso de poder- para reforzar las identidades tradicionalmente excluidas" (Walsh, 2009, p.49). Se busca así fortalecer las identidades culturales de los estudiantes indígenas.

Se concibe que el diálogo de saberes a nivel epistemológico todavía está en proceso (Rist et al, 2004). Tomemos por ejemplo la realidad peruana en el desarrollo de las ciencias ambientales y el enfoque que comprenden estos, o la construcción de una educación ambiental intercultural, a nivel de la Educación Básica y particularmente de la Educación Superior, o de la investigación ambiental y el desarrollo sostenible. Ello es importante de resaltar porque según Orr (1992), toda educación es educación medio-ambiental, si la entendemos como una que busca la protección de la vida humana y de la vida de toda especie existente en el planeta.
A nivel de lo académico global ya se incorporan estudios de la medicina tradicional -Youyou Tu, médico china que estudia, entre otros, el tema de la medicina tradicional china, ganó el premio Nóbel de Medicina en el año $2015^{4}$, y en nuestra universidad ya se invita a estudiosos de este tipo de medicina del Perú (lo vimos en conferencias patrocinadas por la Universidad)-. A nivel de educación primaria se incorporan en las escuelas EIB técnicas agrícolas o pastoriles tradicionales, por ejemplo, con la participación de los sabios y sabias indígenas y se articulan con el área curricular de ciencia y tecnología ; $y$, en educación no formal, organizaciones no gubernamentales enlazan estos saberes o saberes de otros pueblos indígenas con los saberes de la ciencia y tecnología modernas,para la solución de problemas reales de la actividad comunal (productiva, por ejemplo) (conversación con Ingrid Guzmán).

No obstante, llamamos la atención sobre un hecho importante. Todavía en las ciencias ambientales convencionales y en el discurso de desarrollo peruanos se preconiza el antropocentrismo o dominio del hombre sobre otros seres vivos, mientras que las sociedades indígenas, por ejemplo, la andina, que hemos estudiado, postula lo holístico de la red de vida, en la cual el ser humano es parte y no domina la naturaleza (Maurial, 2011). El desarrollo epistémico será intercultural cuando lo académico postule la reciprocidad entre seres humanos y naturaleza como base para que la biodiversidad articulada a la diversidad cultural se sostenga en la agricultura tradicional y en los alimentos nativos y orgánicos.

Asimismo, se afirma que en la realidad andina un acontecimiento o hecho social como una festividad, por ejemplo, es a la vez artístico, económico, educativo, agrícola, ganadero, religioso, social, cultural (Maurial, 2011). Entonces, se tiene el reto de aprender de los pueblos indígenas que nos impela,

4 Veáse: http://www.lavoz.com.ar/ciudadanos/quien-es-tu-youyou-laprimera-mujer-china-en-conseguir-un-premio-nobel 
en lo académico, a romper con la fragmentación disciplinar y desarrollar la interdisciplinariedad y la transdisciplinariedad. La primera:

"Comprende la interacción entre dos o más disciplinas diferentes y ocurre en la intersección entre disciplinas. Esto puede varias desde el compartir de ideas hasta la integración total de conceptos, metodología, procedimientos, teorías, terminología, datos, organización de la investigación y entrenamiento (Natural Sciencies and Engineering Research Council of CanadaNSERC, 2004, citado en Henao et al, 2017, p. 183)."

Apostamos por compartir ideas entre disciplinas. Por su parte la trasndisciplinariedad "no se esfuerza por dominar muchas disciplinas. Su objetivo es abrir todas las disciplinas a que compartan y que observen más allá de ellas" (Henao et al, 2017, p. 183). Es una nueva mirada que no encasille al especialista en su disciplina.

Señalan Rist et al (2004) al respecto de los conceptos recién citados: "Las comunidades epistémicas de las ciencias (se refiere a comunidades que horizontalizan el encuentro entre las ciencias y el conocimiento local) tienen un enorme compromiso con la interdisciplinariedad y están compartiendo e incrementando la comprensión transdisciplinaria de la ciencia" (Rist et al, 2004, p. 18) (traducción libre).

Queda en nuestro presente, como universidad, una realidad de integración inter-facultades que va de la mano con la interculturalización de nuestra universidad. Ello implica el respeto por la diversidad cultural, lingüística y de género de la sociedad peruana y de nuestros estudiantes, y su práctica activa en nuestras aulas.

\section{Referencias bibliográficas}

1. Abarca, G. (2015). Apuntes, Educación y Desarrollo. Recuperado de http://www.unesco.org/new/fileadmin/MULTIMEDIA/FIELD/ Santiago/pdf/APUNTE09-ESP.pdf
2. Henao C. García, D. Aguirre, E. González, A. Bracho, R. Solórzano, J. y Arboleda, A. Multidisciplinariedad, interdisciplinariedad y transdisciplinariedad en la formación para la investigación en ingeniería. Revista Lasallista de Investigación. 2017;14(1):179-197.

3. Hernández Y. Acerca del género como categoría analítica. Nómadas, revista de ciencias sociales y jurídicas. 2006;13(1):1-10.

4. Ishisawa. Notas sobre la gestión del diálogo de saberes. En Diálogo de Saberes. Una aproximación epistemológica. Lima: PRATEC; 2021. URL disponible en: http://www.pratec.org/wpress/pdfs-pratec/ Dialogo-saberes-aproxim-epist.pdf

5. Maurial M. Condiciones de aprendizaje en la escuela multigrado (documento de trabajo). Lima, UNICEF; 2000.

6. Maurial M. Equidad, diversidad y modelos propios: ligando género, etnicidad y educación en Bolivia. En: Sichra, I. (comp). Género, etnicidad y educación en América Latina. Madrid: Morata; 2003:195203.

7. Maurial M. Pintando el ambiente: sobre conocimiento indígena y educación. Cusco: CBC; 2011. (Adaptación de tesis de la autora: Andean rural children's views of the environment: a qualitative study, para optar el grado de Doctor en Filosofía en Teorías y Políticas Educativas, Pennsylvania State University; 2009).

8. MINEDU. Documento Nacional de Lenguas Originarias del Perú. Lima: MINEDU; 2013.

9. MINEDU. Currículo Nacional de la Educación Básica. Lima: MINEDU; 2016.

10. National Research Council. Lost crops of the Incas. Little-known plants of the Andes with promise for worldwide cultivation. Washington, DC: National Academic Press; 1989.

11. Orr D. Ecological literacy: education and the transition to a postmodern world. Albany: State University of New York Press; 1992.

12. Rist S, Zimmermann A and Wiesmann U. From epistemic monoculture to cooperation between epistemic communities -lessons learnt from development research. Centre for Development and Environment and NCCR North-South, University of Berne, Switzerland. Paper presented at the International Conference "Bridging Epistemologies". Millennium Assessment. Alexandria, Egypt (17-20. March, 2004). http://www.millenniumassessment.org/documents/bridging/papers/ rist.stephan.pdf.

13. UNESCO. Nuestra diversidad creativa. Informe de la Comisión Mundial de Cultura y Desarrollo. París: UNESCO; 1996.

14. UNESCO. Declaración Universal sobre la Diversidad Cultural. Una visión una plataforma conceptual un semillero de ideas un paradigma nuevo; 2001. URL disponible en: http://www.educatolerancia.com/ wp-content/uploads/2016/12/DIVERSIDAD-CULTURAL.pdf

16. Walsh C. Interculturalidad, Estado, sociedad: Luchas de-coloniales de nuestra época. Quito: UASB \& Abya Yala; 2009.

17. Zúñiga M. La educación en un contexto bilingüe quechua castellano. Revista Peruana de Ciencias Sociales. 1987; 1.

\section{Correspondencia}

Mahia Maurial MacKee

mahia.maurial.m@upch.pe

Fecha de recepción: 19-08-2021.

Fecha de aceptación:31-08-2021.

Conflicto de interés: ninguno, según la autora.

Financiamiento: por la autora. 